\title{
ORGANIZACIÓN DE LA FORMACIÓN PRÁCTICA DE FUTUROS DOCENTES DE EDUCACIÓN PRIMARIA EN CHILE: ESTUDIO EXPLORATORIO DESDE LAS UNIVERSIDADES
}

\author{
Rosario Rivero ${ }^{1}$, \\ Violeta Arancibia ${ }^{2}$, \\ Susana Claro ${ }^{3}$, \\ Francisco Lagos ${ }^{4}$, \\ Constanza Hurtado ${ }^{5}$
}

\section{RESUMEN}

La experiencia práctica es un componente esencial de la formación de docentes. Sin embargo, la evidencia en Chile respecto de la cantidad, intensidad y calidad de dicho componente práctico es escasa. Usando una metodología mixta, esta investigación cuantifica y analiza la organización de la formación práctica del $70 \%$ de las instituciones que imparten carreras de Pedagogía Básica en Chile. Los resultados muestran, en primera instancia, que el componente práctico se encuentra presente en todas las universidades analizadas, pero existe bastante dispersión en términos de cantidad de cursos, horas y organización de estas experiencias; y, en segundo término, que estas diferencias se encuentran asociadas a características institucionales como, por ejemplo, la acreditación. Tanto los instrumentos como los resultados del estudio contribuyen a este campo, no solo entregando claves para guiar procesos de mejora, sino también para generar indicadores que permitan evaluar la intensidad y calidad del componente práctico de la Formación Inicial Docente (FID), aumentando las exigencias en procesos de acreditación.

Conceptos clave: formación inicial docente, formación práctica, políticas formación inicial docente.

\section{HOW THE PRACTICAL COMPONENT OF INITIAL PRIMARY EDUCATION TEACHER TRAINING IS ORGANIZED IN CHILE'S UNIVERSITIES: AN EXPLORATORY STUDY}

\section{ABSTRACT}

Practical professional experience is a central component of initial teacher training. However, there is a dearth of evidence regarding the quantity, intensity, and quality of this practical component, in Chile. Using a mixed methods approach, this paper quantifies and analyses how

Universidad Diego Portales, Facultad de Educación, Santiago, Chile.

Contacto: rosario.rivero@udp.cl

2 Universidad de los Andes, Santiago, Chile. Contacto: varancib@mac.com

3 Pontificia Universidad Católica de Chile, Escuela de Gobierno, Santiago, Chile. Contacto: sclaro@gmail.com

4 Investigador independiente, Santiago, Chile: Contacto: flagosm@gmail.com

5 Investigadora independiente, Santiago, Chile: Contacto: cehurtad@uc.cl 
this practical component is organized in $70 \%$ of the higher education institutions that train primary school teachers in Chile. The results show, first and foremost, that all universities include a practical component in their curriculum, but there are significant differences among universities in terms of the number of courses, hours, and organization of these experiences, and secondly, these differences are associated with institutional characteristics such as accreditation. This study's instruments and results will contribute to this field, through guiding improvement processes as well as the development of indicators that could be used to evaluate the intensity and quality of the practical component in teacher training programs, ultimately increasing the demands of accreditation processes.

Key concepts: teacher education policies, practical experience, initial teacher education.

\section{Introducción}

La efectividad docente se ha transformado en un elemento clave de la agenda educacional a nivel mundial (Darling-Hammond, 2017). Consecuentemente, la Formación Inicial Docente (en adelante FID) y, en particular, la dimensión de la formación práctica ha adquirido protagonismo. Tanto la literatura nacional como internacional han concluido no solo que las experiencias prácticas dentro de la FID son centrales para el aprendizaje de las habilidades y actitudes propias del futuro docente (Barber \& Mourshed, 2008; Grossman et al., 2009; Montecinos, Barrios y Tapia, 2011; Ortúzar, Flores, Milesi y Cox, 2009; Papay, West, Fullerton \& Kane, 2012; Zeichner, 2010), sino que gran parte del conocimiento pedagógico y profesional se aprende a través de la práctica y de la observación de estas en contextos auténticos de desempeño profesional (Barber \& Mourshed, 2008; Papay et al., 2012 Zeichner, 2010).

Chile no es la excepción. La preocupación por la calidad de los profesores y su formación inicial es creciente. Sucesivas revisiones nacionales e internacionales han enfatizado la necesidad de generar medidas para fortalecer la formación y disminuir la distancia entre la FID chilena y los requerimientos del sistema escolar nacional (Comisión FID, 2005; Consejo Asesor Presidencial, 2006; Organización para la Cooperación y el Desarrollo Económicos, OCDE, 2004; Panel de expertos para una educación de calidad, 2010). Coherentemente con la relevancia de la calidad docente, las políticas nacionales de FID han transitado desde iniciativas de 
14 ORGANIZACIÓN DE LA FORMACIÓN PRÁCTICA DE FUTUROS DOCENTES DE EDUCACIÓN PRIMARIA EN CHILE: ESTUDIO EXPLORATORIO DESDE LAS UNIVERSIDADES R. Rivero, V. Arancibia, S. Claro, F. Lagos, C. Hurtado

apoyo e incentivo, hacia una combinación de apoyo con medidas de rendición de cuentas y mayor regulación en el aseguramiento de la calidad (Cox, Meckes y Bascopé, 2011).

La literatura a nivel nacional es escasa en esta área, pero en los últimos años algunas investigaciones en Chile han profundizado ciertos aspectos de la formación práctica, principalmente estudiando su organización y asociación con percepciones de autoeficacia. En este contexto, las investigaciones chilenas coinciden en señalar aspectos tales como número, progresión y calidad de las prácticas como aspectos relevantes a considerar para evaluar la asociación entre experiencias prácticas y mayor percepción de autoeficacia de docentes novatos.

Por otra parte, la formación práctica ha tenido menos atención desde la política educativa, al no tener medidas de apoyo ni aseguramiento específicas. En la última década, entre las medidas para mejorar la FID en Chile que podrían indirectamente haber incidido en la formación práctica, destacan tres políticas principales: la acreditación de las carreras de Pedagogía en 2007, la implementación de los Convenios de desempeño en 2012 y la Ley de Carrera Docente en 2016. Específicamente, la acreditación tiene como objetivo fundamental certificar la calidad de los programas ofrecidos por las instituciones según los objetivos declarados por estas y por estándares nacionales (Ley No 20.129). Para ello, la Comisión Nacional de Acreditación (CNA) evalúa, interna y externamente, entre otras cosas, el "Perfil de egreso y resultados," donde se señala que los programas deben contar con al menos un semestre de formación práctica (CNA, 2014). Por su parte, los convenios de desempeño consisten en un convenio entre el Ministerio de Educación de Chile (Mineduc) y las instituciones formadoras, el cual financia por tres años cambios orientados al aseguramiento de la formación inicial definidos en un Plan de Mejoramiento Institucional y un Plan de Mejoramiento de Programa. Algunas recomendaciones para las instituciones son diseñar mallas curriculares en torno a prácticas efectivas y estrechar la relación con contextos escolares reales. Por otra parte, la política más reciente, la Ley de Carrera Docente, busca mejorar las condiciones laborales y de formación de quienes ejercen la profesión. Para 
cumplir con el primer objetivo, la ley define nuevos requisitos para la acreditación de los programas de FID, aumentando las exigencias en desempeño mínimo en la PSU para la admisión ${ }^{6}$ y estableciendo convenios con centros escolares para la realización de las prácticas tempranas y progresivas. En resumen, estas normativas, en conjunto, orientan a las instituciones a incluir al menos un semestre de prácticas y mantener vínculos más estrechos con centros escolares y contextos auténticos de aprendizaje.

Así, si bien tanto la investigación como las políticas educativas han reconocido crecientemente la importancia del componente práctico en la FID, hay escasa evidencia empírica respecto de la oferta y organización de la formación práctica en la FID de Chile y, aparentemente, un bajo alineamiento entre las políticas que apuntan a mejorar la FID y el aseguramiento de la calidad de la formación práctica. Este trabajo pretende entregar información en torno a la formación práctica existente en la FID de Pedagogía Básica en Chile y su asociación con las políticas públicas orientadas a su mejoramiento. En particular, este estudio:

- describe el componente de formación práctica disponible en la FID de Chile del año 2016, focalizándose en la cantidad, tipo, actividades e intensidad de este componente reportado por las mismas instituciones; $y$

- compara las características institucionales (años de acreditación, selectividad, y existencia de convenio de desempeño) entre programas FID con cada tipo de componente práctico.

El estudio presenta, en primera instancia, los antecedentes bibliográficos involucrados, luego se introducen los aspectos metodológicos y los resultados de la investigación, para terminar con conclusiones y algunas recomendaciones para el aseguramiento de la calidad de FID.

6 El año 2017 el puntaje mínimo promedio fue de 50\% superior de la distribución de la PSU o bien $30 \%$ superior del ranking. Se estima que para 2023, los programas de Pedagogía serán exclusivos para alumnos con puntajes en el tercio superior de la Prueba de Selección Universitaria, PSU, y del decil superior del ranking de notas de su establecimiento. 
16 ORGANIZACIÓN DE LA FORMACIÓN PRÁCTICA DE FUTUROS DOCENTES DE EDUCACIÓN PRIMARIA EN CHILE: ESTUDIO EXPLORATORIO DESDE LAS UNIVERSIDADES R. Rivero, V. Arancibia, S. Claro, F. Lagos, C. Hurtado

\section{Antecedentes}

\subsection{Marco conceptual: definición de formación práctica}

La FID tiene como principal objetivo entregar habilidades y conocimientos requeridos en el ejercicio profesional a futuros docentes, desarrollando la capacidad de reflexión y análisis teórico, como también las habilidades prácticas. Estas cualidades pueden ser resumidas en tres tipos de conocimiento: declarativo (saber qué), procedural (saber cómo) y condicional (saber cuándo y porqué aplicar ciertos procedimientos), cuya conjugación configura el "conocimiento profesional práctico” (Mattsson, Eilertsen \& Rorrison, 2012).

Para lograr este conocimiento, los distintos programas de FID combinan componentes de formación teórica con los de formación práctica. Actualmente, se discute cómo organizar ambos mecanismos para generar este conocimiento profesional, existiendo dos corrientes fundamentales que Russell (2014) ha denominado "teoría primero, práctica después" y "experiencia primero, comprensión después". La primera corriente, también conocida como la "aproximación de introducción gradual", señala que es necesaria una extensa preparación antes de asumir las responsabilidades docentes en las aulas de los establecimientos educacionales. Esta preparación se adquiriría a través de la observación y colaboración en la sala de clases universitarias, donde los estudiantes de Pedagogía se pueden enfrentar a la complejidad de los procesos de aprendizaje, y desarrollar control sobre sus comportamientos docentes. La segunda corriente, denominada de "inducción rápida", es menos tradicional, y se fundamenta en la idea de que los docentes en formación progresen rápidamente para encargarse de clases completas, siendo esta una experiencia drásticamente distinta de la observación o asistencia de un profesor.

Considerando la realidad chilena, en el marco de este estudio, se definió la formación práctica como toda actividad o experiencia de campo en que el estudiante, durante la etapa de formación inicial docente, se relaciona de manera directa o indirecta con una o más comunidades escolares, a fin de profundizar referentes teóricos o aspectos vocacionales del futuro docente como también 
observar, recolectar información o ejercitar una o más habilidades y conocimientos que serán requeridos en su ejercicio profesional como docente. De esta forma, esta definición abarca las dos corrientes descritas por Russell (2014), incorporando al análisis actividades que se llevan a cabo tanto en el aula universitaria como en centros escolares.

A su vez, en este estudio y siguiendo a Greenberg, Pomerance y Walsh (2011), se distingue la formación práctica en dos tipos principales:

- Experiencias prácticas. Se refieren a las diversas oportunidades tempranas de campo en que los futuros profesores realizan algunas de las siguientes actividades: observan, asisten, trabajan como tutores, preparan material, enseñan y/o investigan, insertos total o parcialmente en una comunidad escolar.

- Prácticas profesionales. Son aquellas pasantías que ofrecen una experiencia final de formación intensiva y extensiva, en la que los futuros docentes desarrollan y ejercitan competencias estando completamente insertos en una comunidad escolar.

\subsection{Evidencia internacional y nacional de los efectos de las prácticas}

Diversos estudios internacionales caracterizan la organización de la formación práctica — extensión, actividades e intensidad de los cursos prácticos durante la FID_, y analizan la relación entre estos elementos y alguna medida de habilidad, conocimiento y/o desempeño de los futuros docentes. Los resultados de estas investigaciones no son concluyentes respecto del impacto de las prácticas en la percepción de preparación y autoeficacia de los futuros docentes, pero pueden ser beneficiosas en relación con el desempeño futuro del docente. Por ejemplo, Ronfeldt y Reininger (2012), a partir de una muestra de respuestas de 1.000 profesores novatos, concluyeron que la duración de la práctica profesional tiene un efecto pequeño en la autopercepción de preparación instruccional y eficacia. Resultados similares se reportan en Gurvitch y Metzler (2009), Ronfeldt (2012), y Ronfeldt, Reininger y Kwok (2013). Por el contrario, Darling-Hammond (2006) consignó que aquellas prácticas organizadas como un trabajo supervisado y que se extienden por al menos 30 semanas, se asocian positivamente a la 
18 ORGANIZACIÓN DE LA FORMACIÓN PRÁCTICA DE FUTUROS DOCENTES DE EDUCACIÓN PRIMARIA EN CHILE: ESTUDIO EXPLORATORIO DESDE LAS UNIVERSIDADES R. Rivero, V. Arancibia, S. Claro, F. Lagos, C. Hurtado

autopercepción de los docentes principiantes. Por otra parte, cuando se analiza la asociación entre la organización de la formación práctica y el desempeño del futuro docente, medida como logro de aprendizaje de los alumnos de los docentes novatos y retención en el sistema, las investigaciones encuentran consistentemente una asociación positiva y significativa entre el aprendizaje de los estudiantes y los créditos orientados al manejo de clases (Harris \& Sass, 2011) y el número de cursos prácticos (Boyd, Grossman, Lankford, Loeb \& Wyckoff, 2009; National Research Council, 2010) y la probabilidad de mantenerse trabajando en el sistema escolar y no abandonar la carrera (Brill \& Macartney, 2008).

En Chile, la evidencia respecto de la oferta de la formación práctica, y su asociación con alguna medida de habilidad, conocimiento o desempeño de los futuros docentes, es escasa y reciente. En el país, esta literatura se ha centrado principalmente en caracterizar la formación práctica de un grupo pequeño de programas FID, estudiando las actividades, extensión e intensidad de los cursos, y la asociación entre estos elementos y las percepciones de los futuros docentes. En un estudio reciente, Montecinos (2016) analizó las mallas curriculares de 10 programas de Pedagogía Básica, evidenciando que algunos de ellos contaban con menos de cuatro cursos prácticos. A su vez, destacó la consistencia de las instituciones en la secuencia de actividades asociadas a cada una de las prácticas, reflejando oportunidades progresivas para observar lo aprendido teóricamente, planificar y evaluar y, para representar habilidades y conocimientos adquiridos. Así mismo, la autora consignó que las instituciones han aumentado el número de cursos prácticos y la progresión de las actividades y que los programas optan por la premisa de "saber teoría para hacer docencia", por sobre la de "aprender haciendo", lo cual no se asocia a un cambio en el paradigma de la relación teoríapráctica. Por último, la investigación destacó el rol del programa de "Fortalecimiento de la Formación Inicial de Profesores" del año 2001 en el aumento de cursos prácticos y su progresión.

Por otra parte, Solís et al. (2011) analizaron la modalidad de acompañamiento a los estudiantes durante los cursos prácticos desde la mirada de los futuros profesores, para 10 carreras de Pedagogía 
Básica. La muestra incluyó respuestas de 1.657 estudiantes (63\% de Pedagogía Básica), distribuidas en práctica inicial (33\%), intermedia (50\%) y final (17\%). Los autores determinaron que las prácticas son guiadas por las universidades y supervisadas por los centros de práctica y resaltan que la modalidad de acompañamiento varía según la práctica que se analiza. A su vez, mostraron que las expectativas de los estudiantes difieren de la realidad, especialmente, en prácticas iniciales e intermedias, ya que ellos esperan mayor participación de los centros de práctica.

Desde un enfoque comparativo, Martinic y otros (2014), a partir de información levantada desde las instituciones, estudiaron el componente de prácticas de Pedagogía Básica de la Pontificia Universidad Católica de Chile en relación con la formación práctica de Medicina en cuatro dimensiones: extensión e intensidad; oportunidades de aprendizaje; relación de la práctica con el currículo; y evaluación. Este estudio resulta interesante ya que la Medicina y la Pedagogía son carreras que, a diferencia de otras, comparten el hecho que la práctica sea clave e intensiva durante el proceso formativo. Los resultados mostraron que las prácticas de Pedagogía representaban el $12 \%$ del currículo de formación y se concentraban en los dos últimos años. En cuanto a las oportunidades de aprendizaje, estas dependían del tipo de práctica, confirmando la tendencia a la progresión. En esta institución, en los cursos iniciales de Pedagogía la actividad central era observar; en los cursos intermedios, los estudiantes se integraban a un centro de práctica para diseñar propuestas acotadas de intervención, las que siempre eran supervisadas; mientras que en la práctica final, los estudiantes asistían a un establecimiento durante cinco jornadas escolares a la semana, con el objetivo de desempeñarse autónomamente. La selección de los centros de práctica se regía por criterios de factibilidad y el trabajo conjunto con la institución se consignó como débil. En la carrera de Medicina, por otro lado, las experiencias prácticas fueron más extensas, como resultado de una mayor centralidad en la formación de la autonomía profesional y mayor relación y regulación con los centros de práctica.

Ahora bien, los estudios nacionales que abordan la percepción de autoeficacia de los futuros profesores muestran hallazgos similares 
20 ORGANIZACIÓN DE LA FORMACIÓN PRÁCTICA DE FUTUROS DOCENTES DE EDUCACIÓN PRIMARIA EN CHILE: ESTUDIO EXPLORATORIO DESDE LAS UNIVERSIDADES R. Rivero, V. Arancibia, S. Claro, F. Lagos, C. Hurtado

a los estudios internacionales. En efecto, Montecinos et al. (2011) analizaron las creencias de autoeficacia de 111 estudiantes de Pedagogía Básica que cursan la práctica profesional. Los autores concluyeron que un estilo de supervisión directiva (versus supervisión reflexiva) se asocia con mayores niveles de autoeficacia cuando el practicante ha realizado tareas estructuradas que requerían de conocimientos instrumentales, así como también, cuando el mentor modeló prácticas de enseñanza rutinaria, con escasa atención a los contenidos y tareas poco desafiantes para sus alumnos. Finalmente, una supervisión más reflexiva se asociaría con mayores niveles de autoeficacia cuando el profesor guía ha modelado prácticas de enseñanza de alta calidad. De manera similar, Avalos y Bascopé (2014) analizaron las respuestas de 54 profesores en formación antes y después de su práctica final. Luego de 16 entrevistas y 12 observaciones de clase dentro de este grupo los resultados indican que la extensión de las prácticas estudiadas variaba entre 11 y 20 semanas, dentro de las cuales se encontraban casos en que el futuro docente solo enseñaba durante un mes, dedicando el resto del tiempo a labores de apoyo rutinarias. De la muestra, un 53\% declaró escaso contacto con otros docentes de los centros de práctica; un 80\% indicó que los mentores los observaron durante un 75\% de ellas; y un 57\% de los entrevistados señaló que sus supervisores universitarios los observaron menos de un $25 \%$ de la práctica. Consistentemente, un tercio calificó como deficiente la supervisión recibida.

Si bien la investigación chilena da luces acerca de la diversidad del componente práctico disponible en la FID de Chile, esta información no necesariamente representa la mayoría de la oferta del país. Durante el 2016 existían 135 programas de FID ofrecidos por 40 instituciones, mientras que el mayor estudio de los descritos anteriormente solo revisó 11 programas. Al mismo tiempo, la información de las investigaciones previas se verificó antes de la última política nacional que ha impactado en el componente práctico de la FID en Chile, por lo que el escenario actual en torno a la organización de la formación práctica disponible en FID puede ser distinta. Por estas razones es indispensable actualizar la información en torno a esta temática, para que sea representativa de una proporción mayor de programas y que aborde con mayor profundización las características 
del componente práctico y su asociación con la política educativa, tal como se pretendió hacer en el presente estudio.

1.3. Políticas nacionales para el aseguramiento de la calidad de la FID

En la última década, diversas políticas nacionales han buscado mejorar y asegurar la calidad de la FID. Si bien directamente no profundizan en el componente de formación práctica, algunas de estas políticas generan orientaciones de mejora que podrían impactar en la organización de dicho componente. Son de especial interés la política de acreditación, la implementación de los convenios de desempeño y la selectividad de las instituciones (considerando las nuevas exigencias en la selección de alumnos de la Ley de Carrera Docente $)^{7}$. A continuación se describen brevemente.

\section{Metodología}

\subsection{Recolección de información y decisiones metodológicas}

Este estudio sigue un diseño mixto secuencial en tres etapas, donde cada una de ellas involucró un método de recolección de datos particular y un conjunto de decisiones metodológicas específicas. Estas etapas convergieron en una etapa final donde se levantó y analizó la información, según se detalla a continuación.

\subsubsection{Primera etapa: definición del universo}

Con el objetivo de definir el universo y decidir cuál era la unidad de análisis más apropiada (instituciones o carreras), se analizó la información de las mallas curriculares publicadas en los sitios web de todos los programas de Pedagogía Básica de Chile (135 programas en 40 universidades). El análisis evidenció que el 60\% de las instituciones tiene una malla equivalente para todos los programas, y que en el 40\% hay diferencias en las mallas según sedes, jornadas, modalidades y/o menciones. De este 40\%, las diferencias entre mallas

Otras políticas que podrían incidir en la formación práctica son el uso de la institución de los Estándares Orientadores y de la Evaluación Inicia, ambos pertenecientes al programa Inicia desarrollado en 2007. 

EDUCACIÓN PRIMARIA EN CHILE: ESTUDIO EXPLORATORIO DESDE LAS UNIVERSIDADES -

se refieren principalmente a cursos teóricos de las distintas menciones y no a cursos de la formación práctica, donde estos últimos son muy similares, por lo que este estudio consideró a las instituciones como la unidad de análisis ${ }^{8}$. El levantamiento de datos a través de la encuesta descrita más adelante se focaliza en los programas de Pedagogía Básica con mayor matrícula de cada institución.

\subsubsection{Segunda etapa: estudio exploratorio de casos}

Se realizaron entrevistas presenciales semiestructuradas a una muestra intencionada de encargados de práctica de ocho instituciones de cuatro regiones ${ }^{9}$ del país. Estas entrevistas exploraron la organización de las prácticas, el tipo de actividades que asocian a cada curso práctico, la extensión y el momento de la formación en que se sitúan y los cambios en los últimos años. Sus respuestas se analizaron por medio de la técnica de matriz de vaciado, método inductivo de análisis de información cualitativa (ver, por ejemplo, Groenland, 2014) y fueron utilizados para la creación de la encuesta descrita en la etapa siguiente.

\subsubsection{Tercera etapa: diseño del instrumento ${ }^{10}$}

Sobre la base de la información de la etapa anterior y de la revisión de literatura en torno a la formación práctica, los estándares de prácticas de otros sistemas educativos y la organización de la formación práctica de ocho programas de FID ${ }^{11}$ en Canadá, Alemania, Holanda,

8 Como se menciona más adelante, los cursos con actividades prácticas que ocurren en el aula universitaria no son considerados como cursos de práctica, por lo que bajo esta definición es posible decir que todos los programas de una institución tienen los mismos cursos prácticos.

9 La mitad de los entrevistados ejercía en sedes de la Región Metropolitana, dos en la Quinta Región de Valparaíso, y los restantes en la Octava Región del Biobío y Décima Región de los Lagos. La mitad de las universidades contaba con convenios de desempeño.

10 La versión final del instrumento puede ser descargada en el siguiente link: https:// centroestudios.mineduc.cl/

11 Esta revisión fue realizada en el marco del proyecto Fonide, que no está incluida en el presente artículo. 
Australia y España ${ }^{12}$, se diseñó una encuesta en línea para medir la organización de las prácticas ${ }^{13}$. Se incluyeron preguntas de selección múltiple y abiertas, y el instrumento fue pretesteado. La Figura 1 sintetiza la información levantada para este estudio. Se cuantificó el número de cursos prácticos, el semestre de realización de estos, el número de horas asociado a cada uno, y se incluyeron los tipos de práctica y las actividades que se llevan a cabo en cada una de ellas.

Entre el aprendizaje recogido de las entrevistas se destaca la priorización de tipos de cursos de práctica. Las personas entrevistadas mostraron tener alta diversidad en lo que era considerado como un curso de práctica, cuyas actividades ocurrían en el aula universitaria en contraste con los cursos de práctica en un centro. Por esta razón se decidió que el estudio cubriría exclusivamente la información relacionada con los cursos cuyas acciones prácticas ocurren en centros. Específicamente se definió que un curso de este tipo será aquel donde "el alumno asiste al aula en un establecimiento educacional a realizar observación, intervención acotada y/o práctica extensa en experiencias de desempeño docente y conducción del proceso de enseñanza-aprendizaje". Expresamente se les dijo a los encargados que al contestar el cuestionario no consideraran aquellos "cursos orientados a la formación de habilidades prácticas que se desarrollan en la universidad".

A partir de información de respuestas abiertas de los encuestados se construyeron cuatro tipos de prácticas: inicial, intermedia 1 , intermedia 2 y profesional:

- Las prácticas iniciales se definen como aquellas en las que los estudiantes realizan, al menos, una de las siguientes actividades: observación, preparación de material o apoyo a profesores.

12 Del total de programas revisados solo en España se trata de programas de pregrado. Los restantes son programas de formación de posgrado que cursan quienes tienen grado de bachiller (Bachelor, B.A.). Los programas corresponden a: Stanford Teaching Certification Program, STEP (2015), Urban Teacher Center, UTC (2015), Teaching Fellows, TNTP (2015), Relay GSE-Teaching Residency (2012), The Developmental Teacher Education Program at UC, Berkeley (2015), entre otros. Para mas información ver Proyecto Fonide.

13 En el marco del proyecto Fonide fueron creadas cinco dimensiones adicionales a las aquí presentadas. 
- Las prácticas intermedias corresponden a aquellas cuyo objetivo es insertarse progresivamente en los establecimientos, analizar reflexivamente la estructura y funcionamiento de una unidad educativa, iniciar al estudiante en el desarrollo de experiencias de aprendizaje, planificar e implementar unidades didácticas de las distintas disciplinas y elaborar instrumentos de evaluación. También, en estas experiencias, los estudiantes deberían relacionarse con docentes u otros profesionales de las escuelas. En general, involucran la colaboración en la planificación, conducción y evaluación de las actividades pedagógica y las intervenciones o experiencias docentes en las clases.

- La práctica intermedia 1 incluye intervenciones concisas o en una clase completa.

- La práctica intermedia 2 implica que los alumnos intervienen en algunas clases o unidad completa, sin ser los responsables del resultado de los estudiantes de ese curso.

- Las prácticas profesionales corresponden a aquellas en las que los estudiantes se responsabilizan del diseño, conducción del proceso de enseñanza aprendizaje de los alumnos, evaluación y sus resultados.

\begin{tabular}{|c|c|}
\hline Dimensión & Indicador \\
\hline Tipo de práctica & Inicial, intermedia y final. \\
\hline Actividades & $\begin{array}{l}\text { Observación. } \\
\text { Preparación de material pedagógico. } \\
\text { Apoyo de profesores. } \\
\text { Intervención acotada en clases. } \\
\text { Intervención en algunas clases. } \\
\text { Intervención en varias clases. } \\
\text { Diseño y conducción del proceso completo enseñanza aprendizaje. }\end{array}$ \\
\hline Temporalidad & Semestre de inicio práctica. \\
\hline Intensidad & $\begin{array}{l}\text { Número de cursos prácticos por año. } \\
\text { Número de cursos prácticos por tipo de práctica. } \\
\text { Porcentaje de instituciones con uno, dos, tres y todos los tipos de } \\
\text { práctica. }\end{array}$ \\
\hline Extensión & $\begin{array}{l}\text { Semanas de duración práctica promedio. } \\
\text { Horas totales dedicadas al componente de prácticas en la carrera. } \\
\text { Horas a la semana en el centro de práctica. } \\
\text { Horas al semestre con supervisor. } \\
\text { Horas al semestre dedicadas a la reflexión con pares. }\end{array}$ \\
\hline
\end{tabular}

Figura 1. Organización de la formación práctica en los programas de FID.

Fuente: Elaboración propia. 
2.1.4. Cuarta etapa: aplicación de la encuesta y análisis de resultados En 2015 (año de aplicación de la encuesta) 72 carreras agrupadas en 40 instituciones recibieron a 1.838 alumnos de primer año para cursar el programa. El promedio de alumnos matriculados en primer año por carrera fue de 25 ; un $75 \%$ se impartía en horario diurno y $72 \%$ contaba con acreditación. Por su parte, ese mismo año existían 135 programas con alumnos cursando algún año de la carrera; 19 de ellos correspondían a institutos profesionales y 88 (65\%) se encontraban acreditados. En los últimos diez años, tanto la matrícula de primer año como la matrícula total de estos programas ha disminuido (64\% y $52 \%$, respectivamente).

Las respuestas obtenidas en la encuesta en línea representan un $70 \%$ de las instituciones (27), las cuales concentraban el $80 \%$ de la matrícula de primer año de estudiantes de Pedagogía Básica de $2015^{14}$. Al caracterizarlas, el $22 \%$ de ellas correspondió a universidades estatales, un $52 \%$ a universidades privadas y un $26 \%$ universidades privadas pertenecientes al CRUCH. Un 44\% de estas instituciones presentó un puntaje de ingreso mínimo promedio (de Lenguaje y Matemáticas) en la PSU inferior a 500 puntos y el 56\% de ellas tuvo puntajes promedio de ingreso superiores a 500 puntos. Un 7\% de estas instituciones no estaba acreditada, un 37\% contaba con dos o tres años de acreditación, y un 56\% con cuatro o más años; un tercio de ellas tenía convenios de desempeño de formación inicial. La mayoría de los programas se impartía exclusivamente en horario diurno (76\%); un $21 \%$ en horario diurno y vespertino; y en un caso se trató de un programa vespertino. La Tabla 1 resume esta información.

14 En 2015 existían 135 programas agrupados en 40 universidades con 10.553 alumnos cursando Pedagogía Básica. En términos de distribución regional, la oferta se concentraba en la Región Metropolitana (42\%) y, en segundo y tercer lugar, en la Región de Biobío y la Región de Valparaíso, respectivamente. El promedio de puntaje de ingreso fue de 479 puntos en la PSU, y solo en un 70\% de las carreras ingresaron exclusivamente alumnos de puntajes promedio superiores a 500 puntos. En promedio, se matricularon 25 alumnos por carrera y $75 \%$ de ellas se impartía en horario diurno (Consejo Nacional de Educación, CNED, 2016). 
26 ORGANIZACIÓN DE LA FORMACIÓN PRÁCTICA DE FUTUROS DOCENTES DE EDUCACIÓN PRIMARIA EN CHILE: ESTUDIO EXPLORATORIO DESDE LAS UNIVERSIDADES R. Rivero, V. Arancibia, S. Claro, F. Lagos, C. Hurtado

Tabla 1

Características de instituciones participantes

\begin{tabular}{llcc}
\hline Características & & Número & Porcentaje \\
\hline \multirow{2}{*}{ Tipo de institución } & Estatal & 6 & $22 \%$ \\
\cline { 2 - 4 } & Privada & 14 & $52 \%$ \\
\cline { 2 - 4 } & Privada CRUCH & 7 & $26 \%$ \\
\hline Selectividad & No & 12 & $44 \%$ \\
\cline { 2 - 4 } & Sí & 15 & $56 \%$ \\
\hline Acreditación & No & 10 & $7 \%$ \\
\cline { 2 - 4 } & Dos o tres años & 15 & $56 \%$ \\
\cline { 2 - 4 } & Cuatro años o más & 18 & $67 \%$ \\
\hline Convenio desempeño de & No & 9 & $33 \%$ \\
\cline { 2 - 4 } formación inicial & Sí & 27 & $100 \%$ \\
\hline Total & & & \\
\hline
\end{tabular}

Fuente: Elaboración propia.

En relación con los 27 encuestados, la mayoría de ellos desempeñaba el rol de encargado de práctica como función principal. El 89\% contaba con cinco o menos años de experiencia en el rol; un $48 \%$ ejercía otro cargo dentro de la misma universidad (docencia, coordinación de acreditación, entre otros) y alrededor de un tercio declaró trabajar adicionalmente fuera del contrato de la universidad. En promedio, los entrevistados declararon 35 horas de contrato semanal de las cuales dedicaban aproximadamente 16 a la labor de coordinación de prácticas. Respecto del número de estudiantes que coordinaban semestralmente, un $24 \%$ de ellos estaba a cargo de coordinar menos de 40 estudiantes, mientras que $28 \%$ coordinaba entre 40 y 80 estudiantes. El resto de los entrevistados coordinaba prácticas semestrales de un número mayor de estudiantes, en algunos casos cerca de 500.

Respecto de los análisis, se realizó estadística descriptiva en relación con la información recolectada de la formación práctica y luego se asoció a características institucionales. Se consideraron cuatro variables:

- Tipo de institución: privadas, públicas y privadas pertenecientes al CRUCH.

- Selectividad: no selectiva (puntaje de ingreso mínimo promedio de Lenguaje y Matemáticas en la PSU inferior a 500 puntos) y selectivas (puntaje superior a 500 puntos). 
- Acreditación: no acreditada, dos a tres años de acreditación y más de cuatro años.

- Convenio de desempeño, distinguiendo a instituciones que no han sido parte del programa de aquellas que sí lo han sido.

\section{Resultados}

3.1. Organización de la formación práctica en universidades chilenas

Los resultados muestran la organización de la formación práctica para las 27 instituciones y sus carreras de Pedagogía Básica. En total, estas cuentan con 147 cursos prácticos, de los cuales 21 corresponden a prácticas iniciales; 27 a prácticas intermedias 1; 52 a prácticas intermedias 2; y 47 a prácticas profesionales, siendo estas dos últimas los principales cursos prácticos realizados por las instituciones. A continuación se describen los resultados.

\subsubsection{Actividades de cada tipo de curso práctico}

En relación con las actividades realizadas en cada tipo de práctica según institución, la Tabla 2 muestra que los alumnos en las prácticas iniciales en casi todas las instituciones realizan observación y apoyo a sus profesores, mientras que el 55\% prepara material pedagógico. Para el caso de las prácticas intermedias 1, las actividades realizadas son muy consistentes entre instituciones, ya que la gran mayoría de ellas procuran que los alumnos aprendan a través de la observación, preparación de material pedagógico, apoyo a profesores e intervención acotada en clases. Las prácticas intermedias 2 se caracterizan por realizar intervenciones en algunas o en varias clases, incorporando las actividades realizadas en prácticas anteriores. Por último, la práctica profesional se diferencia en que todas las instituciones basan el aprendizaje de sus alumnos en que ellos realicen el diseño y conducción del proceso completo de enseñanza y aprendizaje en un curso, al interior de su lugar de práctica. 
28 ORGANIZACIÓN DE LA FORMACIÓN PRÁCTICA DE FUTUROS DOCENTES DE EDUCACIÓN PRIMARIA EN CHILE: ESTUDIO EXPLORATORIO DESDE LAS UNIVERSIDADES R. Rivero, V. Arancibia, S. Claro, F. Lagos, C. Hurtado

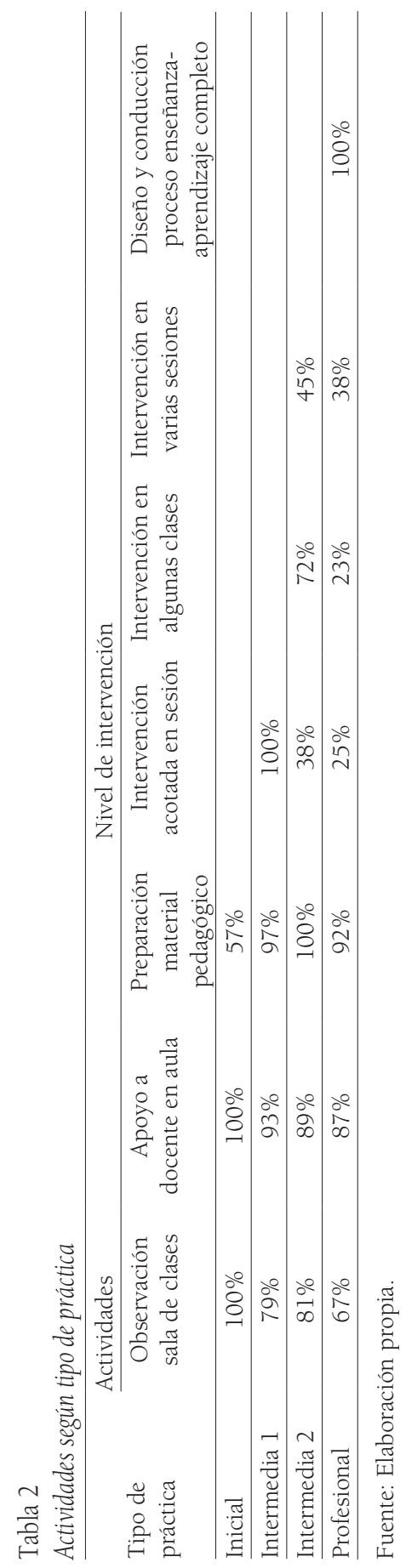




\subsubsection{Intensidad de formación práctica en los programas}

Respecto de la intensidad de la formación práctica en los programas FID, se describe la cantidad de cursos prácticos por institución y por tipo a lo largo de los programas.

La Tabla 3 muestra que el promedio de cursos prácticos ofrecidos a lo largo de la formación docente de las instituciones participantes es de 5,4 cursos. Es decir, —dado que solo se identifica un curso práctico por semestre en todas las instituciones- según la definición amplia usada en este estudio, en promedio, los alumnos tienen algún curso práctico en más del $50 \%$ de los semestres de sus carreras. Sin embargo, la variación entre instituciones es alta, existiendo carreras que solo tienen tres cursos prácticos, mientras que otras presentan cursos prácticos todos o casi todos los semestres de la carrera. En relación con los tipos de cursos prácticos disponibles, se observa una mayor oferta promedio de cursos dedicados a las actividades propias de las prácticas intermedias 2 y profesional (dos cursos en cada caso). Si bien todos los programas reportan, al menos, un curso de práctica profesional, la cantidad de semestres con práctica profesional en la carrera también varía por institución: un $41 \%$ de ellas tiene solo un semestre en toda la carrera; un 33\% tiene dos; y un $20 \%$ tiene tres o cuatro semestres de práctica profesional, evidenciando grandes diferencias en la intensidad de la formación práctica entre las instituciones. Estas diferencias se ilustran en la Figura 2.

Tabla 3

Número de cursos prácticos promedio según tipo de práctica

\begin{tabular}{lccc}
\hline Tipo de práctica & Promedio & Mínimo & Máximo \\
\hline Inicial & 1 & 0 & 2 \\
\hline Intermedia 1 & 1 & 0 & 2 \\
\hline Intermedia 2 & 2 & 0 & 5 \\
\hline Profesional & 2 & 1 & 4 \\
\hline Total & 5,4 & 3 & 9
\end{tabular}

Fuente: Elaboración propia. 
3.1.3. Progresión y temporalidad de cursos prácticos a lo largo de los programas

Para observar la progresión de los cursos prácticos a lo largo de cada FID se reporta la cantidad de tipos de práctica disponible en cada institución y luego la temporalidad de estas en la duración del programa. Para empezar, la Tabla 4 muestra que, en relación con la presencia de cada tipo de curso práctico durante la FID, un 67\% de los casos contempla práctica inicial, un $78 \%$ incluye cursos de práctica intermedia 1 , el $74 \%$ de intermedia 2 y todas las carreras incluyen dentro de su formación práctica, al menos, un curso de práctica profesional. También se observa que solo un tercio de las carreras estudiadas incorpora los cuatro tipos de cursos de práctica antes descritos, mientras que un $55 \%$ de las instituciones omite al menos un curso práctico dentro de la progresión de las actividades prácticas. El detalle en la variedad y diferencias en el número de semestres con cada tipo de práctica por institución se presenta en la Figura 2.

Tabla 4

Distribución tipo de práctica por institución

\begin{tabular}{lcc}
\hline Tipos de práctica & Número & Porcentaje \\
\hline Inicial & 18 & $67 \%$ \\
\hline Intermedia 1 & 21 & $78 \%$ \\
\hline Intermedia 2 & 20 & $74 \%$ \\
\hline Profesional & 27 & $100 \%$ \\
\hline Un tipo de curso práctico & 0 & $0 \%$ \\
\hline Dos tipos de cursos prácticos & 4 & $15 \%$ \\
\hline Tres tipos de cursos prácticos & 15 & $55 \%$ \\
\hline Todos los tipos de cursos prácticos & 8 & $30 \%$
\end{tabular}

Fuente: Elaboración propia. 


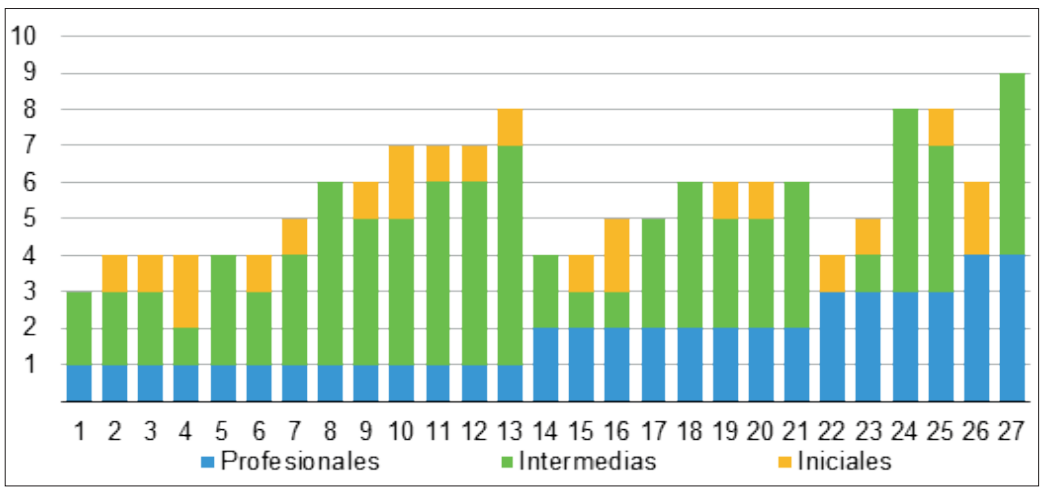

Figura 2. Distribución de instituciones según cantidad de cursos prácticos de cada tipo (número de semestres por tipo de práctica por institución).

Nota: Eje X: Instituciones ordenadas de menor a mayor número de semestres de práctica profesional. Eje Y: Número de semestres.

Fuente: Elaboración propia.

Por su parte, las Tablas 5a y 5b muestran la distribución de los tipos de cursos prácticos por año. Se observa que entre las instituciones que contemplan práctica inicial, estas las sitúan en los primeros dos años, mientras que aquellas que incluyen prácticas intermedias 1 las concentran, principalmente, entre el segundo y tercer año de formación. Los cursos prácticos con actividades de intervención en algunas o varias clases (intermedia 2) se concentran en general en tercer y cuarto año de formación, pero un quinto de las universidades los considera durante el segundo año. En cuanto a la práctica profesional, esta aparece desde el cuarto año, con excepción de dos casos en los que se sitúa en el tercer año.

Complementariamente, Tabla 5b reporta el año y tipo de la primera actividad práctica que ofrece cada programa. Se observa que la mayoría de las universidades sitúa el primer curso práctico en el segundo año (52\%), seguido por aquellas que comienzan el primer año (44\%). 
32 ORGANIZACIÓN DE LA FORMACIÓN PRÁCTICA DE FUTUROS DOCENTES DE EDUCACIÓN PRIMARIA EN CHILE: ESTUDIO EXPLORATORIO DESDE LAS UNIVERSIDADES R. Rivero, V. Arancibia, S. Claro, F. Lagos, C. Hurtado

Tabla $5 \mathrm{a}$

Porcentaje de instituciones que ofrece cada tipo de práctica por año

\begin{tabular}{cccccc}
\hline Año/práctica & Inicial & Intermedia 1 & Intermedia 2 & Profesional & Total \\
\hline 1 & $26 \%$ & $15 \%$ & $0 \%$ & $0 \%$ & 0,8 \\
\hline 2 & $41 \%$ & $33 \%$ & $22 \%$ & $0 \%$ & 1 \\
\hline 3 & $11 \%$ & $37 \%$ & $63 \%$ & $7 \%$ & 1,2 \\
\hline 4 & $0 \%$ & $4 \%$ & $48 \%$ & $74 \%$ & 1,5 \\
\hline 5 & $0 \%$ & $0 \%$ & $4 \%$ & $44 \%$ & 0,5 \\
\hline
\end{tabular}

Fuente: Elaboración propia.

Respecto de la progresión de prácticas durante la formación, se observa que alrededor de la mitad de las instituciones comienza sus cursos prácticos de manera progresiva, correspondiendo el primer curso a práctica tipo inicial en el primer o segundo año de la carrera. Por otro lado, un 45\% de instituciones comienza su formación práctica con cursos prácticos intermedios, ya sea en primer año o más tarde. Esto sugiere que los estudiantes de estos programas cuentan con escasas experiencias iniciales de observación, preparación de material pedagógico e intervenciones acotadas de clases, antes de enfrentarse a situaciones prácticas de mayor complejidad.

Tabla $5 b$

Tipo y año del primer curso práctico en cada institución

\begin{tabular}{cccccc}
\hline \multicolumn{7}{c}{ Instituciones cuyo primer curso práctico fue } & \multirow{2}{*}{ Total } \\
\cline { 1 - 4 } Año & Inicial & Intermedia 1 & Intermedia 2 & Profesional & \\
\hline 1 & 8 & 4 & & & $12(44 \%)$ \\
\hline 2 & 7 & 6 & 1 & $14(52 \%)$ \\
\hline 3 & 1 & & $1(4 \%)$ \\
\hline 4 & & & 0 \\
\hline 5 & $15(55 \%)$ & $11(41 \%)$ & $1(4 \%)$ & 0 \\
\hline Total & & & & 27 \\
\hline
\end{tabular}

Fuente: Elaboración propia.

\subsubsection{Extensión de cada curso de práctica}

Como se ha indicado, parte esencial de la formación práctica de los estudiantes depende de su exposición en los centros de práctica. La Tabla 6 muestra las semanas promedio de los distintos tipos de práctica, así como la cantidad de horas semanales directamente en centros de práctica o en actividades asociadas, como reuniones con 
el supervisor o dedicadas a la reflexión con pares, distinguiendo por tipo de práctica ${ }^{15}$. Como se aprecia, los alumnos dedican entre 12 y 14 semanas a los cursos prácticos, siendo mayores las semanas dedicadas a la práctica profesional, seguida de la intermedia 2 y de la inicial. Las horas semanales son consistentes con la progresión de las actividades y responsabilidades de cada tipo de práctica. Así, en promedio, los programas asignan 5 horas semanales en los centros de práctica en las prácticas iniciales, dedicación que aumenta a 5,7 horas semanales promedio y 7,2 horas semanales, en las prácticas intermedias 1 y 2 , respectivamente. Finalmente, los estudiantes destinan 14 horas promedio a centros de práctica en prácticas profesionales, equivalentes a menos de media jornada laboral. Luego, los coordinadores declaran que en las prácticas iniciales e intermedias los estudiantes dedican, en promedio, entre una y dos horas por semana a reuniones individuales con el supervisor, independientemente del tipo de práctica por el que se pregunta. Finalmente, los estudiantes dedican, en promedio, alrededor de dos horas semanales a reflexionar acerca de las prácticas con pares, sin importar el tipo de curso práctico en el que se encuentren.

Tabla 6

Extensión de la formación práctica según tipo de práctica

\begin{tabular}{lcccc}
\hline & Inicial & Intermedia 1 & Intermedia 2 & Profesional \\
\hline Semanas promedio & 11,9 & 10,9 & 12,6 & 13,1 \\
\hline $\begin{array}{l}\text { Promedio horas semanales en } \\
\text { centro de práctica }\end{array}$ & 5,4 & 5,7 & 7,2 & 14 \\
\hline $\begin{array}{l}\text { Promedio horas semanales con } \\
\text { supervisor }\end{array}$ & 1,5 & 1,9 & 2,4 & 1,7 \\
\hline $\begin{array}{l}\text { Promedio horas semanales } \\
\text { reflexión con pares }\end{array}$ & 2,1 & 2,1 & 2,1 & 2,1 \\
\hline
\end{tabular}

Fuente: Elaboración propia.

Finalmente, se construyó un indicador de horas totales destinadas a los cursos prácticos a lo largo de la formación. Se observa una alta varianza entre universidades (ver Figura 3); el promedio de horas dedicadas a la práctica es de 714 horas, con un mínimo de 48 horas y un máximo de 1.974 horas. Respecto del total de horas asignadas a

15 Estos promedios solo incluyen a quienes respondieron por el tipo de práctica indicado. 

EDUCACIÓN PRIMARIA EN CHILE: ESTUDIO EXPLORATORIO DESDE LAS UNIVERSIDADES R. Rivero, V. Arancibia, S. Claro, F. Lagos, C. Hurtado

actividades prácticas en las universidades de la muestra cabe destacar que, en términos comparados, estos totales se asemejan a lo observado en programas a nivel internacional. Por ejemplo, Jenset, Klette y Hammerness (2018), al estudiar programas de formación docente en Finlandia, Noruega y Estados Unidos mostraron que el promedio de horas destinadas a experiencias prácticas en los programas analizados era de 625 horas, con un mínimo de 432 horas y un máximo de 1.000 horas $^{16}$. Así, si bien esto no necesariamente se relaciona con la calidad de este componente práctico, al menos da una indicación respecto de cómo se compara descriptivamente con experiencias en otros países.

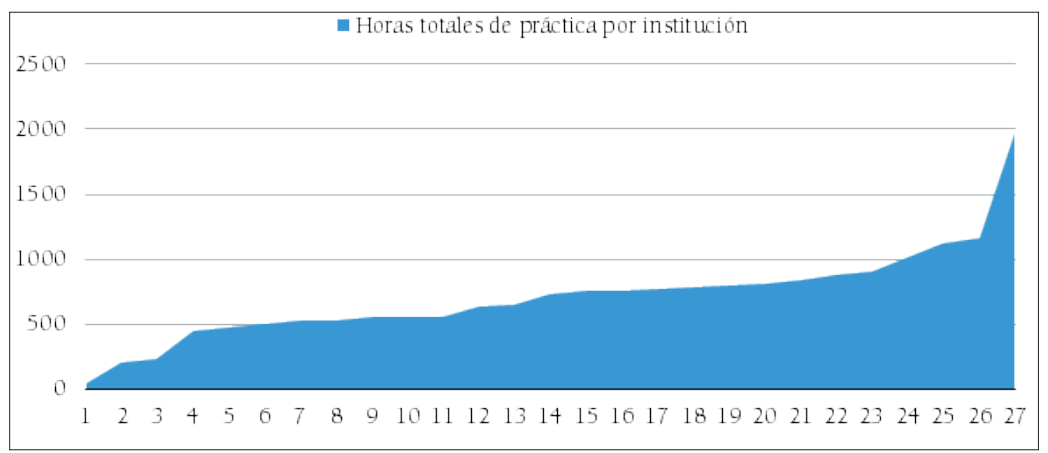

Figura 3. Distribución de instituciones según las horas totales de práctica incluidas en su programa. Horas totales promedio: 714 horas.

Nota: Eje X: Instituciones ordenadas de menor a mayor número de horas totales de práctica incluida en el programa. Eje Y: Número de horas.

Fuente: Elaboración propia.

\subsection{Asociación entre la formación práctica y características de las instituciones formadoras}

Los siguientes resultados muestran diferencias y similitudes en los modos en que instituciones formadoras con distintas características -tipo, selectividad, acreditación, y si cuenta con convenio de desempeño de formación inicial— organizan la formación práctica de Pedagogía Básica.

16 Tal como se mencionó previamente este artículo no incluye el análisis de programas a nivel internacional llevado a cabo como parte del proyecto Fonide. Sin embargo, cabe destacar que en dicho análisis de experiencias en Estados Unidos, Canadá, Alemania, Países Bajos, Australia y España el rango de horas totales destinadas a experiencias prácticas es similar al observado aquí, con un máximo de 1.500 horas. El estudio completo puede ser descargado desde el centro de documentación del Centro de Estudios Mineduc, http://cedoc.mineduc.cl 
En relación con el número de cursos prácticos, la Tabla 7 muestra que las instituciones privadas del CRUCH presentan un curso práctico promedio más que las instituciones estatales y privadas que no pertenecen a dicho órgano (cuyos promedios son de cinco cursos prácticos a lo largo de la formación). Asimismo, las universidades selectivas superan en un curso práctico a las no selectivas, y aquellas con más años de acreditación superan en un curso a las que pertenecen a la categoría media según años de acreditación (dos o tres años) y a las no acreditadas. Una tendencia similar, pero menor, se encuentra en la organización de las prácticas de universidades que han participado o no de los convenios de desempeño de formación inicial (5,4 versus 5,9 cursos en promedio).

Al comparar por tipo de práctica se encuentra que, las universidades estatales presentan un promedio mayor de cursos de práctica inicial, mientras las privadas del $\mathrm{CRUCH}$ se destacan por más cursos promedio de los tipos intermedia 2 y práctica profesional. Luego en términos de selectividad, las universidades selectivas presentan mayores cursos promedio de prácticas iniciales y prácticas con actividades más avanzadas (intermedia 2 y profesional). En el caso de la acreditación, aquellas universidades con cuatro o más años sobresalen por más cursos en todos los tipos, con excepción de la práctica final, donde las acreditadas por dos o tres años presentan más cursos (1,5 versus 2,1). Finalmente, las facultades que han firmado convenios de desempeño de formación inicial, se distinguen por una mayor concentración de prácticas más avanzadas en comparación con las que no las tienen. En general, al comparar por estos criterios, las universidades con mejores indicadores (mayor selectividad, acreditación y participantes de los convenios) tienden a distribuir los cursos en los cuatro tipos, privilegiando los cursos prácticos que incluyen intervenciones en clases (acotadas o integrales), por sobre las experiencias centradas en la observación o generación de material pedagógico.

La Tabla 8 presenta los promedios de cursos prácticos por año, comparando por características institucionales. Las universidades privadas (pertenecientes o no al CRUCH) muestran promedios mayores en todos los años al comparar con las estatales, con excepción del primero, donde estas últimas las superan. Como ya 
se ha constatado, esto se debe a una mayor inclusión de prácticas iniciales en estas instituciones. Por su parte, las selectivas reportan un promedio mayor durante el primer año, al compararlas con las no selectivas. Una hipótesis posible en torno a esta diferencia, es que las instituciones que forman a alumnos de menor desempeño escolar destinan mayor tiempo a cursos de nivelación en otras áreas (escritura y comprensión lectora, entre otras). En términos de acreditación, las de mayor acreditación presentan mayores promedios desde el segundo año, pese a que las no acreditadas resaltan por tener más cursos en los últimos años, esto debe ser interpretado con precaución, ya que se trata solo de dos casos en la muestra. No hay diferencias entre universidades con o sin convenio de desempeño de formación inicial, con excepción de un promedio de cursos mayor en el segundo año.

Tabla 7

Número de cursos prácticos promedio según tipo de práctica y características institucionales Variables institución Inicial Intermedia 1 Intermedia 2 Profesional Total

\begin{tabular}{lllllll}
\hline \multirow{2}{*}{ Tipo } & Estatal & 1,3 & 0,9 & 1,3 & 1,9 & 5,3 \\
\cline { 2 - 7 } & Privada & 0,6 & 1,1 & 1,7 & 1,8 & 5,1 \\
\cline { 2 - 7 } & Privada CRUCH & 0,7 & 1,0 & 2,7 & 2,1 & 6,6 \\
\hline \multirow{2}{*}{ Selectividad } & No & 0,7 & 1,0 & 1,4 & 1,9 & 5,0 \\
\cline { 2 - 7 } & Sí & 0,9 & 1,0 & 2,2 & 1,9 & 5,9 \\
\hline Acreditación & No & 0,9 & 1,3 & 1,5 & 1,6 & 5,3 \\
\cline { 2 - 7 } & Dos o tres años & 0,7 & 0,7 & 2,1 & 2,1 & 5,6 \\
\cline { 2 - 7 } & Cuatro o más & 1,0 & 2,0 & 2,0 & 1,5 & 6,5 \\
\hline Convenios de \\
\cline { 2 - 7 } $\begin{array}{l}\text { desempeño de } \\
\text { formación inicial }\end{array}$ & Sí & 0,8 & 1,2 & 1,8 & 1,6 & 5,4 \\
\hline \multicolumn{2}{l}{} & 0,9 & 0,6 & 2,0 & 2,4 & 5,9 \\
\hline
\end{tabular}

Fuente: Elaboración propia.

En cuanto al semestre de comienzo de la primera práctica, las universidades con mayor acreditación sobresalen por incluir cursos prácticos durante el primer año $(60 \%)$, a diferencia de la tendencia a postergar al segundo año el comienzo del componente de prácticas, en el grupo de dos a tres años de acreditación. El grupo de no acreditadas comienza en su totalidad en el segundo semestre. En cuanto a los convenios de desempeño de formación inicial, la mayoría de las instituciones con convenios vigentes en 2015 comenzaron las prácticas entre el primer y el tercer semestre (89\% de los casos). 
Tabla 8

Número de cursos prácticos según año y características institucionales

\begin{tabular}{|c|c|c|c|c|c|c|}
\hline & & Año 1 & Año 2 & Año 3 & Año 4 & Año 5 \\
\hline \multirow{3}{*}{ Tipo } & Estatal & 0,7 & 1 & 1,2 & 1,5 & 0,5 \\
\hline & Privada & 0,4 & 1,1 & 1,6 & 1,8 & 0,4 \\
\hline & Privada CRUCH & 0,3 & 1,6 & 1,7 & 1,7 & 1,3 \\
\hline \multirow{2}{*}{ Selectividad } & No & 0,3 & 1 & 1,5 & 1,7 & 0,7 \\
\hline & Sí & 0,5 & 1,3 & 1,4 & 1,7 & 0,8 \\
\hline \multirow{3}{*}{ Acreditación } & No & 1 & 1,5 & 2 & 2 & 0 \\
\hline & Dos o tres años & 0,2 & 0,9 & 1,4 & 1,7 & 0,8 \\
\hline & Cuatro años o más & 0,5 & 1,3 & 1,5 & 1,7 & 0,6 \\
\hline \multirow{2}{*}{$\begin{array}{l}\text { Convenios de desempeño } \\
\text { de formación inicial }\end{array}$} & No & 0,4 & 1,1 & 1,5 & 1,7 & 0,5 \\
\hline & Sí & 0,3 & 1,4 & 1,6 & 1,7 & 0,9 \\
\hline
\end{tabular}

Fuente: Elaboración propia.

La Tabla 9 presenta la distribución por tipos de práctica, considerando las características institucionales y excluyendo la práctica final. Se observan diferencias que indicarían que las instituciones estatales excluyen, en mayor medida, los cursos de práctica intermedia 2 , mientras las privadas omiten en mayor medida las prácticas iniciales. Consistentemente con lo observado antes, al compararlas con las selectivas las que no lo son omiten en su diseño las prácticas iniciales. En el caso de la acreditación, las universidades acreditadas por dos o tres años se caracterizan por contar con una mayor proporción de casos que incluyen cursos de prácticas iniciales e intermedia 1 , si se las compara con las de mayor acreditación y estas últimas, privilegiarían los cursos de práctica intermedia 2. Por su parte, hay más universidades sin convenio de desempeño de formación inicial que incluyen la práctica intermedia 2, al compararlas con las que sí han sido beneficiarias de esta política. Este resultado sugiere que, si bien instituciones selectivas y con convenios de desempeño tienen más prácticas (en promedio), dentro del grupo existe diversidad respecto de la inclusión de todos los tipos de prácticas.

Por otra parte, en relación con la ausencia de gradualidad de las actividades prácticas en la formación en algunos programas del país, se observa que son las privadas no selectivas, y las de mayor acreditación las que omiten con mayor probabilidad la práctica inicial. Esto tiende a compensarse con la inclusión de más cursos 
38 ORGANIZACIÓN DE LA FORMACIÓN PRÁCTICA DE FUTUROS DOCENTES DE EDUCACIÓN PRIMARIA EN CHILE: ESTUDIO EXPLORATORIO DESDE LAS UNIVERSIDADES R. Rivero, V. Arancibia, S. Claro, F. Lagos, C. Hurtado

con actividades denominadas aquí de práctica intermedia 2. Si bien estos casos iniciarían el componente de prácticas con actividades más avanzadas, esto podría explicarse por una concepción que opta por una formación teórica previa a la práctica. 


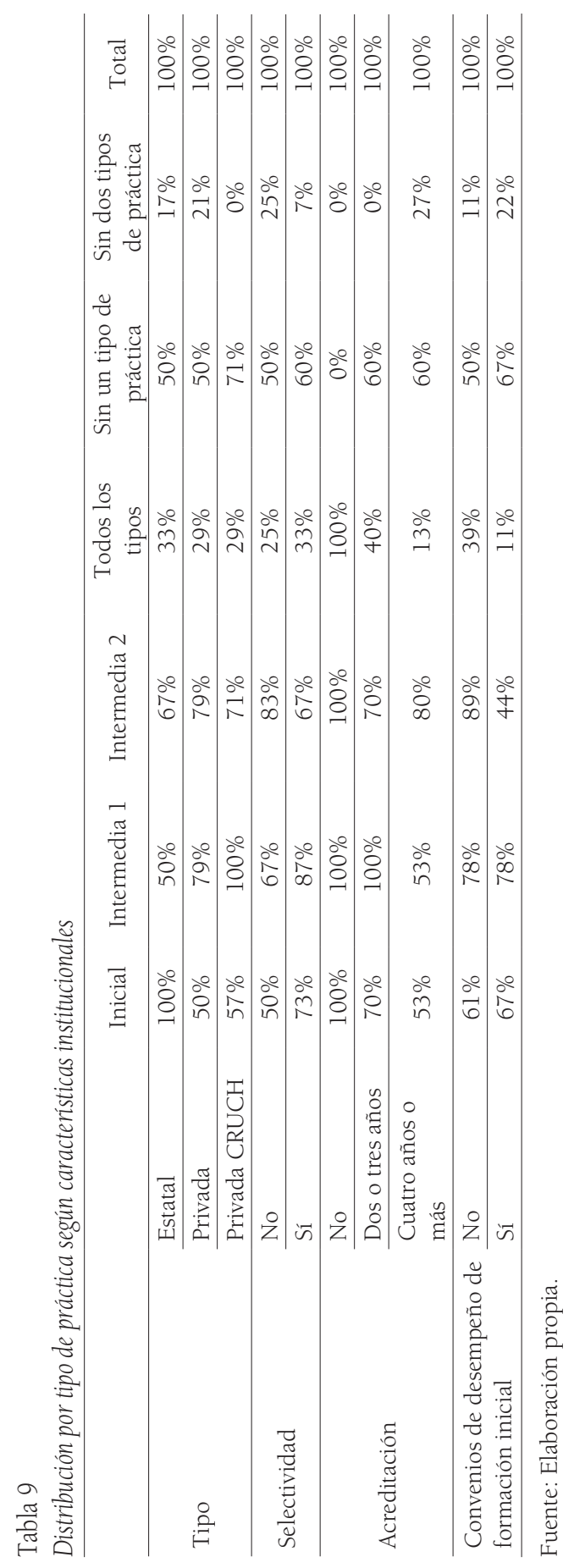


40 ORGANIZACIÓN DE LA FORMACIÓN PRÁCTICA DE FUTUROS DOCENTES DE EDUCACIÓN PRIMARIA EN CHILE: ESTUDIO EXPLORATORIO DESDE LAS UNIVERSIDADES R. Rivero, V. Arancibia, S. Claro, F. Lagos, C. Hurtado

Tabla 10

Extensión práctica profesional y características institucionales

\begin{tabular}{|c|c|c|c|c|c|}
\hline Características & Categorías & Semanas & $\begin{array}{c}\text { Horas } \\
\text { semanales en } \\
\text { el centro }\end{array}$ & $\begin{array}{c}\text { Horas } \\
\text { semanales con } \\
\text { supervisor }\end{array}$ & $\begin{array}{c}\text { Horas } \\
\text { semanales de } \\
\text { reflexión con } \\
\text { pares }\end{array}$ \\
\hline \multirow{3}{*}{ Tipo } & Estatal & 13,1 & 15,0 & 1,5 & 1,5 \\
\hline & Privada & 13,1 & 13,2 & 1,8 & 2,4 \\
\hline & $\begin{array}{l}\text { Privada } \\
\text { CRUCH }\end{array}$ & 12,7 & 14,5 & 1,6 & 2,0 \\
\hline \multirow{2}{*}{ Selectividad } & No & 13,0 & 11,7 & 1,8 & 2,4 \\
\hline & Sí & 13,1 & 15,5 & 1,6 & 1,8 \\
\hline \multirow{3}{*}{ Acreditación } & No & 13,5 & 21,0 & 2,0 & 2,0 \\
\hline & $\begin{array}{l}\text { Dos o tres } \\
\text { años }\end{array}$ & 12,1 & 11,5 & 1,4 & 2,2 \\
\hline & $\begin{array}{l}\text { Cuatro } \\
\text { años o más }\end{array}$ & 13,7 & 14,9 & 1,9 & 2,0 \\
\hline \multirow{2}{*}{$\begin{array}{l}\text { Convenios de } \\
\text { desempeño de } \\
\text { formación inicial }\end{array}$} & No & 12,7 & 13,5 & 1,8 & 2,2 \\
\hline & Sí & 13,8 & 15,0 & 1,3 & 1,7 \\
\hline Total & & 13,1 & 14 & 1,7 & 2,1 \\
\hline
\end{tabular}

Fuente: Elaboración propia.

La Tabla 10 muestra la extensión del curso de práctica profesional en función de las características institucionales. En cuanto a las semanas promedio dedicadas a la práctica profesional, no se encuentran diferencias entre instituciones, ubicándose todas en torno a las 13 semanas (correspondientes a alrededor de tres meses). Aun así, con una semana promedio de diferencia, las universidades privadas pertenecientes al CRUCH, las acreditadas por dos a tres años, y aquellas que no participaron de la política de convenios de desempeño de formación inicial, tienen una semana menos que sus respectivos grupos de comparación. En cuanto a las horas semanales en los centros de práctica, se observan diferencias a favor de las universidades estatales (versus privadas) y selectivas (versus no selectivas). Aquellas de mayor acreditación presentan tres horas adicionales promedio en relación con las de menor acreditación y, aunque con una diferencia menor, las participantes de los convenios de desempeño de formación inicial superan a las que no. Las horas dedicadas al tiempo con supervisores es similar entre los grupos, y las dedicadas a reflexión con pares es levemente mayor en las privadas (no del CRUCH), las no selectivas y las que no tuvieron convenios. 
Finalmente, la Tabla 11 muestra los promedios de horas dedicados a la formación práctica según características institucionales. Son las instituciones privadas del CRUCH, las selectivas, las de mayor acreditación y aquellas que sí cuentan con convenio de desempeño las que destinan más horas a esta formación, al comparar con el promedio de horas y con sus respectivas contrapartes.

Tabla 11

Promedio horas totales dedicadas a prácticas según características institucionales

\begin{tabular}{|c|c|c|c|}
\hline & & $\begin{array}{c}\text { Horas totales de } \\
\text { práctica en la carrera }\end{array}$ & $\begin{array}{l}\text { Categoría respecto } \\
\text { del promedio }\end{array}$ \\
\hline \multirow{3}{*}{ Tipo } & Estatal & 704 & Bajo \\
\hline & Privada & 652 & Bajo \\
\hline & Privada CRUCH & 847 & Sobre \\
\hline \multirow{2}{*}{ Selectividad } & No & 619 & Bajo \\
\hline & $\overline{\text { Sí }}$ & 790 & Sobre \\
\hline \multirow{3}{*}{ Acreditación } & No & 481 & Bajo \\
\hline & Dos o tres años & 614 & Bajo \\
\hline & Cuatro años o más & 812 & Sobre \\
\hline \multirow{3}{*}{$\begin{array}{l}\text { Convenios de } \\
\text { desempeño de } \\
\text { formación inicial }\end{array}$} & No & 635 & Bajo \\
\hline & Sí & 872 & Sobre \\
\hline & Total & 714 & \\
\hline
\end{tabular}

Fuente: Elaboración propia.

\section{Discusión y conclusiones}

Este estudio caracteriza la organización de las prácticas de programas de Pedagogía Básica, a partir del análisis de un cuestionario aplicado a encargados de práctica del $70 \%$ de las universidades que imparten programas de Pedagogía Básica en Chile y que concentran el 80\% de los estudiantes de esta carrera del país. Si bien los resultados alcanzados no permiten establecer una asociación entre los indicadores estudiados y la calidad del componente de prácticas —la cual, entre otros, depende no solo de las instituciones formadoras, sino también de los establecimientos educacionales en los que se desarrollan las experiencias prácticas-la indagación sistemática en torno a dicho componente entrega evidencia de interés para la discusión a nivel nacional. 
En primer lugar, los resultados muestran una alta valoración de las prácticas como eje de los currículos de formación inicial, con más de 700 horas en promedio de formación práctica durante la carrera. Estos resultados están alineados, en general, con lo que señalan las experiencias internacionales. Por ejemplo, Darling-Hammond (2006) considera que aquellas prácticas organizadas como un trabajo supervisado y que se extienden por al menos 30 semanas se asocian positivamente con la autopercepción de los docentes. Sin embargo, existe bastante heterogeneidad en su implementación: la mayoría de las universidades no incluye cursos prácticos durante el primer año de formación; solo un tercio de las instituciones incluye los cuatro tipos de práctica (iniciales, intermedias 1 y 2 y profesionales), dándole una progresión gradual a la experiencia práctica; y casi la mitad de las instituciones solo considera un semestre de práctica profesional. Finalmente, un grupo reducido de carreras (cinco) no alcanza las 500 horas de formación práctica durante toda la carrera, considerando desde los cursos de práctica inicial (observaciones, mayoritariamente) a práctica profesional.

¿Han influido en el aumento de las prácticas en el currículo de formación inicial las políticas educativas que desde el 2008 han promovido estos cambios? Las características institucionales y aquellas derivadas de políticas de aseguramiento de la calidad de la FID, como la acreditación y los convenios de desempeño de formación inicial, se relacionan parcialmente con la variación de los indicadores de organización de las experiencias prácticas. Por ejemplo, las universidades privadas del CRUCH presentan mayor promedio de cursos prácticos a lo largo del programa FID que sus pares estatales y privados no CRUCH. Las instituciones de mayor acreditación, las selectivas y aquellas con convenio de desempeño superan en promedio en un curso de práctica a sus contrapartes y comienzan antes sus prácticas, coincidiendo con las recomendaciones de la literatura especializada. Otro resultado consistente son las horas totales dedicadas a las prácticas, donde las universidades de mayor acreditación y con convenios de desempeño destinan mayor tiempo a esta dimensión. Sin embargo, solo las primeras destacan por un diseño de práctica gradual, que incluye todos los tipos aquí descritos. 
Al considerar las diferencias y similitudes institucionales, este estudio aporta evidencia preliminar respecto del rol que han jugado las políticas orientadas a mejorar los procesos de formación docente (acreditación y convenios de desempeño) en el énfasis que ha adquirido el componente práctico en la FID en Chile.

En términos de las implicancias de este estudio, los resultados aquí expuestos pueden ser claves para guiar procesos de mejora del componente práctico de las FID a nivel institucional y, además, generar indicadores que permitan evaluar la intensidad y calidad de este componente en instituciones formadoras de docentes en Chile, aumentando las exigencias en procesos de acreditación.

En una profesión basada en el desempeño, se adquieren las habilidades fundamentalmente practicando, tal como ocurre en por ejemplo en Medicina. Sin embargo, habitualmente ello no se percibe de esta manera: los típicos programas de formación de profesores raramente dan la oportunidad de practicar las habilidades de forma constante y suficiente como para adquirir maestría y dominio. El tema, en este caso, no es la falta de tiempo, sino la decisión de la mayoría de los programas de enfatizar la teoría por sobre la práctica. Sin embargo, en el último tiempo se aprecian algunos cambios importantes en la formación de profesores en Chile.

Los resultados de este estudio muestran que si bien existen instituciones con una organización adecuada de su formación práctica, un porcentaje importante de ellas no cuenta con todos los tipos de cursos de formación práctica, además de destinar pocas horas y comenzar con ella a mediados de la carrera, evidenciando la presencia de carreras orientadas por modelos "saber la teoría, para ejercer docencia”. Coincidentemente con la revisión de literatura internacional estos modelos de formación tradicionales pueden no ser idóneos, en tanto es posible que no estén dando oportunidad a profesores novatos a desarrollar a cabalidad las competencias requeridas para el ejercicio de la profesión docente. Los resultados de este estudio pueden establecer un punto de comparación para universidades y programas con menor intensidad de sus componentes de práctica, guiando así procesos de mejora en esta línea. 
44 ORGANIZACIÓN DE LA FORMACIÓN PRÁCTICA DE FUTUROS DOCENTES DE EDUCACIÓN PRIMARIA EN CHILE: ESTUDIO EXPLORATORIO DESDE LAS UNIVERSIDADES R. Rivero, V. Arancibia, S. Claro, F. Lagos, C. Hurtado

Por otro lado, para avanzar en el aseguramiento de la calidad de la FID, los resultados no solo entregan una guía respecto de elementos a considerar al momento de evaluar la intensidad, coherencia y profundidad del componente práctico en instituciones formadoras de docentes, sino que también sugieren que es necesario ampliar requerimientos de las prácticas en la acreditación, actualmente reducidos a su existencia y progresión (Montecinos, 2016).

Particularmente, los resultados de este estudio sugieren avanzar en especificar al menos para la práctica profesional, horas mínimas de docencia directa, número de cursos en los que el practicante debe enseñar, niveles de enseñanza, horas semanales que se deben enseñar y tipos de tareas. Para lograr esta mejora es necesario un acompañamiento de recursos, tal como lo establecen los convenios de desempeño de formación inicial, que han mostrado ser una política efectiva para aumentar la intensidad de la formación práctica de las carreras. Este esfuerzo curricular debe acompañarse impulsando nuevas competencias para los supervisores y coordinadores de práctica y avanzar con ello en la definición y gestión de centros de práctica adecuados.

El sistema de desempeño profesional docente, aprobado como ley en 2016, establece explícitamente la necesidad de que el sistema de acreditación incorpore convenios de colaboración con establecimientos educacionales para la realización de prácticas tempranas y progresivas de los estudiantes de Pedagogía, lo cual ha sido una de las dificultades que han enfrentado algunas universidades para realizar sus prácticas. Por lo tanto, el actual criterio de evaluación, referente a la dimensión perfil de egreso y que señala que los programas deben contar con al menos un semestre de formación práctica (CNA, 2014) deberá actualizarse y exigir una mayor cantidad de prácticas.

Este estudio deja abiertas múltiples preguntas para futuras investigaciones. Entre ellas, una de las más relevante es investigar el efecto de los distintos tipos de práctica aquí retratados y su vinculación, por un lado, con las necesidades formativas de los estudiantes que ingresan a estudiar Pedagogía y, por otro, con la 
efectividad, retención y distribución de los egresados en el aula en los distintos tipos de establecimientos educacionales.

Conocer la relación entre cantidad, extensión y calidad de las prácticas y la calidad de la enseñanza de los futuros docentes es un insumo valioso no solo para las universidades, sino también para el diseño de políticas más efectivas en torno a la mejora de calidad docente.

\section{Referencias}

Avalos, B. \& Bascopé, M. (2014). Future teacher trajectory research: Its contribution to teacher education and policy. Education as Change, 18(sup1), S19-S32. https://doi.org/10.1080/16823206.2013.877353

Barber, M. \& Mourshed, M. (2007). How the world's best-performing schools systems come out on top. Recuperado de https://www.mckinsey.com/ industries/social-sector/our-insights/how-the-worlds-best-performingschool-systems-come-out-on-top

Boyd, D. J., Grossman, P. L., Lankford, H., Loeb, S., \& Wyckoff, J. (2009). Teacher preparation and student achievement. Educational Evaluation and Policy Analysis, 31(4), 416-440. https://doi. org/10.3102/0162373709353129

Brill, S. \& McCartney, A. (2008). Stooping the revolving door: Increasing teacher retention. Politics and Policy, 36(5), 750-774. https://doi. org/10.1111/j.1747-1346.2008.00133.x

Comisión FID (2005). Informe Comisión sobre formación inicial docente. Santiago de Chile: Organización de Estados Iberoamericanos para la Educación, la Ciencia y la Cultura, OEI.

Comisión Nacional de Acreditación, CNA. (2014). Criterios de evaluación para carreras y programas de pregrado. Santiago de Chile: Autor.

Consejo Asesor Presidencial (2006). Informe final del Consejo Asesor Presidencial para la Calidad de la Educación. Santiago de Chile: Autor.

Consejo Nacional de Educación, CNED. (2016). Acreditación de carreras de Pedagogía.

Cox, C., Meckes, L., y Bascopé, M. (2010). La institucionalidad formadora de profesores en Chile en la década del 2000: velocidad del mercado y parsimonia de las políticas. Revista Pensamiento Educativo, 46, 205245. Recuperado de http://pensamientoeducativo.uc.cl/index.php/pel/ article/view/468 
46 ORGANIZACIÓN DE LA FORMACIÓN PRÁCTICA DE FUTUROS DOCENTES DE EDUCACIÓN PRIMARIA EN CHILE: ESTUDIO EXPLORATORIO DESDE LAS UNIVERSIDADES R. Rivero, V. Arancibia, S. Claro, F. Lagos, C. Hurtado

Darling-Hammond, L. (2006). Constructing 21st- Century Teacher Education. Journal of Teacher Education, 57(3), 300-314. https://doi. org/10.1177/0022487105285962

Darling-Hammond, L. (2017). Teacher education around the world: What can we learn from international practice? European Journal of Teacher Education, 40(3), 291-309. https://doi.org/10.1080/02619768.2017. 1315399

Greenberg, J., Pomerance, L., \& Walsh, K. (2011). Student teaching in the United States. National Council on Teacher Quality. Recuperado https://www.nctq. org/dmsView/Student_Teaching_United_States_NCTQ_Report

Groenland, E. A. (2014). Employing the matrix method as a tool for the analysis of qualitative research data in the business domain. International Journal of Business and Globalisation, 21(1), 119-134. https://doi.org/10.1504/IJBG.2018.094106

Grossman, P., Compton, C., Igra, D., Ronfeldt, M., Shahan, E., \& Williamson, P. (2009). Teaching practice: A cross-professional perspective. The Teachers College Record, 111(9), 2055-2100. Recuperado de https:// psycnet.apa.org/record/2010-26203-001

Gurvitch, R. \& Metzler, M. W. (2009). The effects of laboratory-based and field-based practicum experience on pre-service teachers' selfefficacy. Teaching and Teacher Education, 25(3), 437-443. https://doi. org/10.1016/j.tate.2008.08.006

Harris, D. N. \& Sass, T. R. (2011). Teacher training, teacher quality and student achievement. Journal of Public Economics, 95(7-8), 798-812. https://doi.org/10.1016/j.jpubeco.2010.11.009

Jenset, I. S., Klette, K., \& Hammerness, K. (2018). Grounding teacher education in practice around the world: An examination of teacher education coursework in teacher education programs in Finland, Norway, and the United States. Journal of Teacher Education, 69(2), 184-197. https://doi.org/10.1177/0022487117728248

Ley No 20.129 (2006). Sistema Nacional de Aseguramiento de Calidad de la Educación

Ley de Carrera Docente (2016). Sistema de Desarrollo Profesional Docente.

Martinic, S., Moreno, R., Müller, M., Pimentel, F., Rittershaussen, S., Calderón, M., \& Cabezas, H. (2014). Análisis comparativo del componente de práctica en el currículo de formación profesional de médicos y profesores en la Pontificia universidad Católica de Chile. Estudios Pedagógicos (Valdivia), 40(1), 179-196. https://doi.org/10.4067/s071807052014000100011 
Mattsson, M., Eilertsen, T. V., \& Rorrison, D. (Eds.) (2012). A practicum turn in teacher education (Vol. 6). Springer Science \& Business Media. https://10.1007/978-94-6091-711-0

Montecinos, C. (2016). Fortalecimiento del currículo de formación práctica en las carreras de pedagogía en Chile: ¿Qué señala la evidencia empírica? En J. Manzi y R. M. García (Eds.), Abriendo las puertas del aula: transformación de las prácticas docentes (pp. 585-614). Santiago de Chile: Ediciones UC.

Montecinos, C., Barrios, C., y Tapia, M. F. (2011). Relación entre estilos de supervisión durante la práctica profesional y las creencias de autoeficacia de los estudiantes de Pedagogía en Educación Básica. Perspectiva Educacional, 50(2), 96-122. Recuperado de http:// www.perspectivaeducacional.cl/index.php/peducacional/article/ viewFile/42/22

National Research Council (2010). Preparing teachers: Building evidence for sound policy. Washington, DC.: Autor.

Organización para la Cooperación y el Desarrollo Económicos, OCDE. (2004). Revisión de Políticas Nacionales de Educación: Chile. Paris: Autor.

Ortúzar, M. S., Flores, C., Milesi, C., y Cox, C. (2009). Aspectos de la formación inicial de docentes y su influencia en el rendimiento académico de los alumnos. Santiago de Chile: Centro de Políticas Públicas, UC.

Panel de Expertos para una Educación de Calidad (2010). Propuestas para fortalecer la profesión docente en el sistema escolar chileno. Santiago de Chile: Ministerio de Educación de Chile.

Papay, J. P., West, M. R., Fullerton, J. B., \& Kane, T. J. (2012). Does an urban teacher residency increase student achievement? Early evidence from Boston. Educational Evaluation and Policy Analysis, 34(4), 413-434. https://doi.org/10.3102/0162373712454328

Ronfeldt, M. (2012). Where should student teachers learn to teach? Effects of field placement school characteristics on teacher retention and effectiveness. Educational Evaluation and Policy Analysis, 34(1), 3-26. https://doi.org/10.3102/0162373711420865

Ronfeldt, M. \& Reininger, M. (2012). More or better student teaching? Teaching and Teacher Education, 28(8), 1091-1106. https://doi.org/10.1016/j. tate.2012.06.003

Ronfeldt, M., Reininger, M., \& Kwok, A. (2013). Recruitment or preparation? Investigating the effects of teacher characteristics and student teaching. Journal of Teacher Education, 64(4), 319-337. https://doi. org/10.1177/0022487113488143 
48 ORGANIZACIÓN DE LA FORMACIÓN PRÁCTICA DE FUTUROS DOCENTES DE EDUCACIÓN PRIMARIA EN CHILE: ESTUDIO EXPLORATORIO DESDE LAS UNIVERSIDADES R. Rivero, V. Arancibia, S. Claro, F. Lagos, C. Hurtado

Russell, T. (2014). La práctica en la formación de profesores: tensiones y posibilidades en la experiencia de aprender a enseñar. Estudios Pedagógicos (Valdivia), 40(especial), 223-238. https://doi.org/10.4067/ s0718-07052014000200013

Solís, M. C., Núñez, C., Contreras, I., Rittershaussen, S., Montecinos, C., \& Walker, H. (2011). Condiciones de la formación práctica de los futuros profesores. Estudios Pedagógicos (Valdivia), 37(1), 127-147. https://doi.org/10.4067/s0718-07052011000100007

Stanford Teacher Education Program (2015). Stanford Graduate School of Education. Recuperado de https://gse-step.stanford.edu

The New Teacher Project, TNTP. (2015). TNTP Teaching Fellows. Recuperado de http://tntpteachingfellows.org

UC Berkeley. (2015). Developmental Teacher Education. Recuperado de http://gse.berkeley.edu/cognition-development/dte

UC Berkeley. (2012). DTE Program Scope and Sequence. Recuperado de http://gse.berkeley.edu/sites/default/files/docs/DTE_courses_0.pdf

Urban Teacher Center, UTC. (2015a). About us: Urban Teacher Center. Recuperado de http://www.urbanteacbout-us/history-and-vision

Urban Teacher Center, UTC (2015b). Our Program: Urban Teacher Center. Recuperado de http://www.urbanteachercenter.org/our-program/fourstep-guarantee

Varas, M. (2008). El valor de una tradición. En P. Felmer (Ed.), Una ventana al mundo. ¿Cómo se forman los profesores de Enseñanza Básica para enseñar Matemática? (pp. 31-59). Santiago de Chile: Academia Chilena de Ciencias.

Zeichner, K. (2010). Rethinking the connections between campus courses and field experiences in college-and university-based teacher education. Journal of Teacher Education, 61(1-2), 89-99. https://doi. org/10.1177/0022487109347671

Recibido:27/02/2019

Aceptado:03/06/2019 